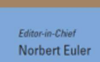

Norbert Euler

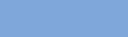

\section{Journal of Nonlinear Mathematical} Physics

ISSN (Online): 1776-0852 ISSN (Print): 1402-9251

Journal Home Page: https://www.atlantis-press.com/journals/jnmp

\title{
Properties of the zeros of the sum of three polynomials
}

Francesco Calogero

To cite this article: Francesco Calogero (2013) Properties of the zeros of the sum of three polynomials, Journal of Nonlinear Mathematical Physics 20:4, 469-474, DOI: https://doi.org/10.1080/14029251.2013.868259

To link to this article: https://doi.org/10.1080/14029251.2013.868259

Published online: 04 January 2021 


\title{
LETTER TO THE EDITOR
}

\section{Properties of the zeros of the sum of three polynomials}

\author{
Francesco Calogero \\ Dipartimento di Fisica, Universitá di Roma “La Sapienza”, Italy \\ Istituto Nazionale di Fisica Nucleare, Sezione di Roma, Italy \\ francesco.calogero@roma1.infn.it,francesco.calogero@uniroma1.it
}

Received 7 June 2013

Accepted 11 September 2013

\begin{abstract}
A system of algebraic equations satisfied by the zeros of the sum of three polynomials are reported.
Keywords: zeros of polynomials, solvable systems of nonlinear partial differential equations

2000 Mathematics Subject Classification: 30C15, 35Exx
\end{abstract}

\section{Introduction}

In a previous paper we highlighted certain properties of the zeros of the sum of two polynomials [6]. In the last section ("Outlook") of that paper we wrote that the "extension of these findings to the zeros of the sums of more than two polynomials will be reported in subsequent papers if these results will be deemed sufficiently novel and neat to justify their publication." In this paper we indeed report the extension of some of those findings to the sum of three polynomials.

Let $p_{N_{j}}^{(j)}(z), j=1,2,3$, be 3 arbitrary (for definiteness, monic) polynomials of the independent (complex) variable $z$, of degree $N_{j}$ with, for definiteness and simplicity, $N_{1} \leq N_{2}<N_{3}$; and let us define as follows the, also monic, polynomial $\psi_{N}\left(z ; t_{1}, t_{2}\right)$ of degree $N \equiv N_{3}$ in $z$ and depending on the 2 parameters $t_{1}$ and $t_{2}$ :

$$
\psi_{N}\left(z ; t_{1}, t_{2}\right)=p_{N}^{(3)}(z)+t_{2} p_{N_{2}}^{(2)}(z)+t_{1} p_{N_{1}}^{(1)}(z) .
$$

Here and hereafter $N$ is an arbitrary positive integer (say, larger than unity, to avoid that some of the formulas written below become too degenerate and trivial).

Hereafter we denote as $z_{n} \equiv z_{n}\left(t_{1}, t_{2}\right)$ the $N$ (generally complex) zeros of this polynomial:

$$
\psi_{N}\left(z ; t_{1}, t_{2}\right)=\prod_{n=1}^{N}\left[z-z_{n}\left(t_{1}, t_{2}\right)\right]
$$

For simplicity, we hereafter assume these $N$ zeros to be all different among themselves; the formulas displayed below remain generally valid when this assumption is violated, but possibly only after taking an appropriate limit.

In this paper we report a system of algebraic equations satisfied by these $N$ zeros $z_{n}\left(t_{1}, t_{2}\right)$. A solvable system of $N$ nonlinear second-order partial differential equations (PDEs) satisfied by these 


\section{F. Calogero}

$N$ functions of two variables is also identified; and it is shown that it belongs to a known class of "Cintegrable" PDEs in multidimensions [2,7]. In the following Section 2 these findings are displayed, and they are then proven in Section 3. A terse Section 4 mentions possible extensions of these findings.

\section{Results}

Hereafter we denote as $W(f ; g)$ the wronskian of the two (monic) polynomials $f(z)$ and $g(z)$,

$$
W(f ; g) \equiv f^{\prime}(z) g(z)-g^{\prime}(z) f(z)
$$

Here and hereafter appended primes denote differentiations with respect to the variable $z$. Of course $W\left(p_{N_{j}}^{(j)} ; p_{N_{k}}^{(k)}\right)$ is a polynomial of degree $N_{j}+N_{k}-1$ if $N_{j} \neq N_{k}$ (and then $W\left(p_{N_{j}}^{(j)} ; p_{N_{k}}^{(k)}\right) /\left(N_{j}-N_{k}\right)$ is monic); while if instead $N_{j}=N_{k}$, then $W\left(p_{N_{j}}^{(j)} ; p_{N_{k}}^{(k)}\right)$ is a (generally not monic) polynomial of degree $N_{j}+N_{k}-2$.

Proposition 2.1. The $N$ zeros $z_{n} \equiv z_{n}\left(t_{1}, t_{2}\right)$ of the polynomial $\psi_{N}\left(z ; t_{1}, t_{2}\right)$, see (1.1) and (1.2), satisfy the following system of $N$ nonlinear algebraic equations:

$$
\begin{array}{r}
\sum_{j=1,2,3 \bmod (3)}\left\{3\left[p_{N_{j}}^{(j) \prime \prime}\left(z_{n}\right) w_{j+1, j+2}\left(z_{n}\right)\right] .\right. \\
\cdot \sum_{\ell=1, \ell \neq n, m \neq n, \ell \neq m}^{N}\left[\left(\frac{1}{z_{n}-z_{\ell}}\right)\left(\frac{1}{z_{n}-z_{m}}\right)\right] \\
-2 p_{N_{j}}^{(j) \prime \prime \prime}\left(z_{n}\right) w_{j+1, j+2}\left(z_{n}\right) \sum_{\ell=1, \ell \neq n}^{N}\left(\frac{1}{z_{n}-z_{\ell}}\right) \\
\left.+\left[p_{N_{j}}^{(j) \prime \prime \prime}\left(z_{n}\right) w_{j+1, j+2}^{\prime}\left(z_{n}\right)\right]\right\}=0, \quad n=1, \ldots, N,
\end{array}
$$

where

$$
w_{j, k}(z) \equiv W\left(p_{N_{j}}^{(j)}(z), p_{N_{k}}^{(k)}(z)\right), \quad j, k=1,2,3 \bmod (3)
$$

Note that, for notational simplicity, we omitted to display explicitly in (2.2a) the dependence of the $N$ zeros $z_{k} \equiv z_{k}\left(t_{1}, t_{2}\right), k=1, \ldots, N$, on the two parameters $t_{1}$ and $t_{2}$. And of course, above and hereafter, the notation $f^{\prime}\left(z_{n}\right)$ denotes the $z$-derivative of the function $f(z)$ evaluated at $z=z_{n} \equiv$ $z_{n}\left(t_{1}, t_{2}\right)$.

Proposition 2.2. The $N$ zeros $z_{n} \equiv z_{n}\left(t_{1}, t_{2}\right)$ of the polynomial $\psi_{N}\left(z ; t_{1}, t_{2}\right)$, see (1.1) and (1.2), satisfy the following system of $N$ nonlinear second-order hyperbolic PDEs,

$$
z_{n, t_{1} t_{2}}=\sum_{\ell=1, \ell \neq n}^{N}\left(\frac{z_{n, t_{1}} z_{\ell, t_{2}}+z_{n, t_{2}} z_{\ell, t_{1}}}{z_{n}-z_{\ell}}\right), n=1, \ldots, N,
$$

provided the boundary conditions $z_{n}(t, 0)$ and $z_{n}(0, t)$ are assigned so that $z_{n}(t, 0)$ are the $N$ zeros of the polynomial $\psi_{N}(z ; t, 0)=p_{N}^{(3)}(z)+t p_{N_{2}}^{(2)}(z)$ and $z_{n}(0, t)$ are the $N$ zeros of the polynomial 


$$
\begin{aligned}
\psi_{N}(z ; 0, t)=p_{N}^{(3)}(z)+t & p_{N_{1}}^{(1)}(z): \\
& \psi_{N}(z ; t, 0)=p_{N}^{(3)}(z)+t p_{N_{2}}^{(2)}(z)=\prod_{n=1}^{N}\left[z-z_{n}(t, 0)\right], \\
& \psi_{N}(z ; 0, t)=p_{N}^{(3)}(z)+t p_{N_{1}}^{(1)}(z)=\prod_{n=1}^{N}\left[z-z_{n}(0, t)\right] .
\end{aligned}
$$

Note that again, for notational simplicity, we omitted to display explicitly in (2.3) the dependence of the $N$ zeros $z_{k} \equiv z_{k}\left(t_{1}, t_{2}\right), k=1, \ldots, N$, on the two parameters $t_{1}$ and $t_{2}$. And of course, above and hereafter, the subscripted variables $t_{1}$ and $t_{2}$ denote partial differentiations with respect to these variables, $z_{n, t_{1}} \equiv z_{n, t_{1}}\left(t_{1}, t_{2}\right) \equiv \partial z_{n}\left(t_{1}, t_{2}\right) / \partial t_{1}, z_{n, t_{2}} \equiv z_{n, t_{2}}\left(t_{1}, t_{2}\right) \equiv \partial z_{n}\left(t_{1}, t_{2}\right) / \partial t_{2}$, $z_{n, t_{1} t_{2}} \equiv z_{n, t_{1} t_{2}}\left(t_{1}, t_{2}\right) \equiv \partial^{2} z_{n}\left(t_{1}, t_{2}\right) / \partial t_{1} \partial t_{2}$.

Via the change of variables

$$
z_{n}\left(t_{1}, t_{2}\right)=\varphi_{n}(x, t), \quad t_{1}=x-c t, t_{2}=x+c t ; n=1, \ldots, N,
$$

the system of PDEs (2.3) becomes

$$
\varphi_{n, t t}-c^{2} \varphi_{n, x x}=\sum_{\ell=1, \ell \neq n}^{N}\left(\frac{\varphi_{n, t} \varphi_{\ell, t}-c^{2} \varphi_{n, x} \varphi_{\ell, x}}{\varphi_{n}-\varphi_{\ell}}\right), n=1, \ldots, N
$$

which has the interesting feature of being invariant under (two-dimensional) Lorentz transformations. It is moreover clear that this system of nonlinear PDEs is a neat extension of the standard "goldfish" model [4,1,3,5], to which the system (2.6) indeed reduces for $c=0$ (and likewise the system (2.3) for $t_{1}=t_{2}=t$, implying $N_{1}=N_{2}, p_{N_{1}}^{(1)}(z)=p_{N_{2}}^{(2)}(z)$; see (2.4)). Clearly this system, (2.6), is a special case of the systems of C-integrable PDEs introduced in [2] and investigated (including the display of solutions in the guise of animations) in [7].

\section{Proofs}

To prove Proposition 2.1 we start from the well-known observation that, up to its normalization and an appropriate identification of the 2 parameters $t_{1}$ and $t_{2}$ (both specified above, see (1.1)), the polynomial $\psi_{N}\left(z ; t_{1}, t_{2}\right)$ can be identified as the solution of the third-order ODE

$$
\operatorname{det}\left(\begin{array}{llll}
\psi_{N}^{\prime \prime \prime}\left(z ; t_{1}, t_{2}\right) & p_{N}^{(3) \prime \prime \prime}(z) & p_{N_{2}}^{(2) \prime \prime \prime}(z) & p_{N_{1}}^{(1) \prime \prime \prime}(z) \\
\psi_{N}^{\prime \prime}\left(z ; t_{1}, t_{2}\right) & p_{N}^{(3) \prime \prime}(z) & p_{N_{2}}^{(2) \prime \prime}(z) & p_{N_{1}}^{(1) \prime \prime}(z) \\
\psi_{N}^{\prime}\left(z ; t_{1}, t_{2}\right) & p_{N}^{(3) \prime}(z) & p_{N_{2}}^{(2) \prime}(z) & p_{N_{1}}^{(1) \prime}(z) \\
\psi_{N}\left(z ; t_{1}, t_{2}\right) & p_{N}^{(3)}(z) & p_{N_{2}}^{(2)}(z) & p_{N_{1}}^{(1)}(z)
\end{array}\right)=0
$$

or equivalently

$$
\begin{array}{r}
a_{3}(z) \psi_{N}^{\prime \prime \prime}\left(z ; t_{1}, t_{2}\right)-a_{2}(z) \psi_{N}^{\prime \prime}\left(z ; t_{1}, t_{2}\right) \\
+a_{1}(z) \psi_{N}^{\prime}\left(z ; t_{1}, t_{2}\right)-a_{0}(z) \psi_{N}\left(z ; t_{1}, t_{2}\right)=0
\end{array}
$$

where (see (2.1))

$$
\begin{gathered}
a_{3}(z)=\sum_{j=1,2,3 \bmod (3)}\left[p_{N_{j}}^{(j) \prime \prime}(z) W\left(p_{N_{j+1}}^{(j+1)}(z), p_{N_{j+2}}^{(j+2)}(z)\right)\right], \\
\text { Co-published by Atlantis Press and Taylor \& Francis } \\
\text { Copyright: the authors }
\end{gathered}
$$


F. Calogero

$$
\begin{aligned}
& a_{2}(z)=\sum_{j=1,2,3 \bmod (3)}\left[p_{N_{j}}^{(j) \prime \prime \prime}(z) W\left(p_{N_{j+1}}^{(j+1)}(z), p_{N_{j+2}}^{(j+2)}(z)\right)\right], \\
& a_{1}(z)=\sum_{j=1,2,3 \bmod (3)}\left[p_{N_{j}}^{(j) \prime \prime \prime}(z) W^{\prime}\left(p_{N_{j+1}}^{(j+1)}(z), p_{N_{j+2}}^{(j+2)}(z)\right)\right], \\
& a_{0}(z)=\sum_{j=1,2,3 \bmod (3)}\left[p_{N_{j}}^{(j) \prime}(z) W\left(p_{N_{j+1}}^{(j+1) \prime \prime}(z), p_{N_{j+2}}^{(j+2) \prime \prime}(z)\right)\right] .
\end{aligned}
$$

At $z=z_{n} \equiv z_{n}\left(t_{1}, t_{2}\right)$ (where $\psi_{N}\left(z ; t_{1}, t_{2}\right)$ vanishes, see (1.2)) formula (3.1b) clearly entails that

$$
\begin{gathered}
a_{3}\left(z_{n}\right) \psi_{N}^{\prime \prime \prime}\left(z_{n} ; t_{1}, t_{2}\right)-a_{2}\left(z_{n}\right) \psi_{N}^{\prime \prime}\left(z_{n} ; t_{1}, t_{2}\right) \\
+a_{1}\left(z_{n}\right) \psi_{N}^{\prime}\left(z_{n} ; t_{1}, t_{2}\right)=0, \quad n=1, \ldots, N .
\end{gathered}
$$

Here and below, for notational simplicity, we often omit to display explicitly the dependence of the zeros $z_{n} \equiv z_{n}\left(t_{1}, t_{2}\right)$ on the two parameters $t_{1}$ and $t_{2}$; as we already did in the preceding section.

On the other hand logarithmic $z$-differentiation of (1.2) implies

$$
\psi_{N}^{\prime}\left(z ; t_{1}, t_{2}\right)=\psi_{N}\left(z ; t_{1}, t_{2}\right) \sum_{\ell=1}^{N}\left(\frac{1}{z-z_{\ell}}\right)
$$

a second $z$-differentiation yields (via (3.4a) and a neat cancellation of the terms featuring double poles)

$$
\psi_{N}^{\prime \prime}\left(z ; t_{1}, t_{2}\right)=\psi_{N}\left(z ; t_{1}, t_{2}\right) \sum_{\ell, m=1, \ell \neq m}^{N}\left[\left(\frac{1}{z-z_{\ell}}\right)\left(\frac{1}{z-z_{m}}\right)\right],
$$

and a third $z$-differentiation yields (again via (3.4a) and a neat cancellation of the terms featuring double poles)

$$
\begin{array}{r}
\psi_{N}^{\prime \prime \prime}\left(z ; t_{1}, t_{2}\right)=\psi_{N}\left(z ; t_{1}, t_{2}\right) . \\
\sum_{\ell, m, k=1,}^{N}\left[\left(\frac{1}{z-z_{\ell}}\right)\left(\frac{1}{z-z_{m}}\right)\left(\frac{1}{z-z_{k}}\right)\right] .
\end{array}
$$

Hence

$$
\begin{aligned}
\frac{\psi_{N}^{\prime \prime}\left(z ; t_{1}, t_{2}\right)}{\psi_{N}^{\prime}\left(z ; t_{1}, t_{2}\right)}= & \sum_{\ell, m=1, \ell \neq m}^{N}\left[\left(\frac{1}{z-z_{\ell}}\right)\left(\frac{1}{z-z_{m}}\right)\right]\left\{\sum_{n=1}^{N}\left(\frac{1}{z-z_{n}}\right)\right\}^{-1}, \\
\frac{\psi_{N}^{\prime \prime \prime}\left(z ; t_{1}, t_{2}\right)}{\psi_{N}^{\prime}\left(z ; t_{1}, t_{2}\right)}= & \sum_{\ell, m, k=1, \ell \neq m, m \neq k, k \neq \ell}^{N}\left[\left(\frac{1}{z-z_{\ell}}\right)\left(\frac{1}{z-z_{m}}\right)\left(\frac{1}{z-z_{k}}\right)\right] . \\
& \cdot\left\{\sum_{n=1}^{N}\left(\frac{1}{z-z_{n}}\right)\right\}^{-1} .
\end{aligned}
$$


And clearly for $z=z_{n} \equiv z_{n}\left(t_{1}, t_{2}\right)$ these formulas yield (for $n=1, \ldots, N$ )

$$
\begin{gathered}
\frac{\psi_{N}^{\prime \prime}\left(z_{n} ; t_{1}, t_{2}\right)}{\psi_{N}^{\prime}\left(z_{n} ; t_{1}, t_{2}\right)}=2 \sum_{\ell=1, \ell \neq n}^{N}\left(\frac{1}{z_{n}-z_{\ell}}\right) . \\
\frac{\psi_{N}^{\prime \prime \prime}\left(z_{n} ; t_{1}, t_{2}\right)}{\psi_{N}^{\prime}\left(z_{n} ; t_{1}, t_{2}\right)}=3 \sum_{\ell=1, \ell \neq n, m \neq n, \ell \neq m}^{N}\left[\left(\frac{1}{z_{n}-z_{\ell}}\right)\left(\frac{1}{z_{n}-z_{m}}\right)\right] .
\end{gathered}
$$

Hence the insertion of these formulas in (3.3) (divided by $\psi_{N}^{\prime}\left(z_{n} ; t_{1}, t_{2}\right)$ ) yields the equation

$$
\begin{array}{r}
3 a_{3}\left(z_{n}\right) \sum_{\ell=1, \ell \neq n, m \neq n, \ell \neq m}^{N}\left[\left(\frac{1}{z_{n}-z_{\ell}}\right)\left(\frac{1}{z_{n}-z_{m}}\right)\right] \\
-2 a_{2}\left(z_{n}\right) \sum_{\ell=1, \ell \neq n}^{N}\left(\frac{1}{z_{n}-z_{\ell}}\right)+a_{1}\left(z_{n}\right)=0, \quad n=1, \ldots, N,
\end{array}
$$

and via the first 3 formulas (3.2) one then gets (2.2). Proposition 2.1 is thereby proven.

To prove Proposition 2.2 we start from the obvious observation that the polynomial $\psi_{N}\left(z ; t_{1}, t_{2}\right)$, see (1.1), satisfies the PDE

$$
\psi_{N, t_{1} t_{2}}\left(z ; t_{1}, t_{2}\right)=0
$$

Let us recall that here and below subscripted variables denote partial differentiations, $\psi_{N, t_{1} t_{2}} \equiv$ $\partial^{2} \psi / \partial t_{1} \partial t_{2}$

We then note that (logarithmic, partial) $t_{1}$-differentiation of (1.2) yields

$$
\psi_{N, t_{1}}\left(z ; t_{1}, t_{2}\right)=-\psi_{N}\left(z ; t_{1}, t_{2}\right) \sum_{n=1}^{N}\left(\frac{z_{n, t_{1}}}{z_{n}-z_{\ell}}\right),
$$

and a (partial) $t_{2}$-differentiation of this formula yields (after a neat cancellation of the double-pole terms, and an appropriate renaming of dummy indices), the formula

$$
\begin{gathered}
\psi_{N, t_{1} t_{2}}\left(z ; t_{1}, t_{2}\right)=\psi_{N}\left(z ; t_{1}, t_{2}\right) \sum_{n=1}^{N}\left\{\left(z-z_{n}\right)^{-1} .\right. \\
\left.\cdot\left[-z_{n, t_{1} t_{2}}+\sum_{\ell=1, \ell \neq n}^{N}\left(\frac{z_{n, t_{1}} z_{\ell, t_{2}}+z_{n, t_{2}} z_{\ell, t_{1}}}{z_{n}-z_{\ell}}\right)\right]\right\} .
\end{gathered}
$$

Note that again, for notational simplicity, in the right-hand side of this formula we omitted to display the dependence of the zeros $z_{n} \equiv z_{n}\left(t_{1}, t_{2}\right)$ on the two parameters $t_{1}$ and $t_{2}$. And clearly this formula, via (3.8), implies (2.3). While the formulas (2.4) are an easy consequence of (1.1). Proposition 2.2 is thereby proven.

\section{Outlook}

A natural extension of these results is to treat a linear combination of an arbitrary number of polynomials. Other interesting extensions may obtain by replacing polynomials with trigonometric polynomials or other combinations of seed functions, and/or replacing the parameters $t_{k}$ with 
functions of (a smaller number of) other parameters. And of course other, potentially interesting, findings may emerge from the investigation of isochronous variants of the solvable systems (2.3) and (2.6) —obtained via the, by now well-known, "trick" generating such systems, see for instance Section 2.1 (entitled "The trick") and Chapter 7 (entitled "Isochronous PDEs") of [5]—and from further investigations of the application of this development to the more general class of solvable systems considered in [2] and [7].

\section{References}

[1] F. Calogero, Motion of poles and zeros of special solutions of nonlinear and linear partial differential equations, and related "solvable" many-body problems, Nuovo Cimento 43B (1978) 177-241.

[2] F. Calogero, A class of C-integrable PDEs in multidimensions, Inverse Problems 10 (1994) 1231-1234.

[3] F. Calogero, Classical many-body problems amenable to exact treatments, Lecture Notes in Physics Monograph m66 (Springer, Berlin, 2001).

[4] F. Calogero, The "neatest" many-body problem amenable to exact treatments (a "goldfish"?), Physica $\mathbf{D}$ 152-153 (2001) 78-84.

[5] F. Calogero, Isochronous systems (Oxford University Press, Oxford, 2008; an updated paperback edition has been published by OUP in September 2012).

[6] F. Calogero, Properties of the zeros of the sum of two polynomials, J. Nonlinear Math. Phys. 20 (2013) $348-354$.

[7] F. Calogero and M. Sommacal, Solvable nonlinear evolution PDEs in multidimensional space, SIGMA 2 (2006) 088 (17 pages); nlin.SI/0612019. 